\title{
NUMERICAL SIMULATION OF THERMAL ENERGY STORAGE IN UNDERGROUND SOIL HEAT ACCUMULATOR
}

\author{
Ján KORTIŠ ${ }^{1, *}$, Michal GOTTWALD ${ }^{2}$ \\ ${ }^{1}$ Department of Structural Mechanics, Faculty of Civil Engineering, University of Žilina, Univerzitná \\ 8215/1, 01026 Žilina. \\ ${ }^{2}$ Research Centre, University of Žilina, Univerzitná 8215/1, 01026 Žilina. \\ corresponding author: jan.kortis@fstav.uniza.sk.
}

\section{Abstract}

The alternative energy sources have been getting popular for last decades as a new way to obtain enough energy especially for countries which do not have rich natural reservoirs of fossil fuels. Gathering the thermal energy from the solar radiation seems to be as one of the cheapest alternatives of them. The disadvantage of it is the overflow of the heat energy during the summer and lack of them during the winter, when the demand for heat is on top. The underground thermal energy storage can be a good alternative for accumulating the heat energy and then offers it on demand. However, it is difficult to monitor the real physical condition in the soil. In the article, the results of numerical simulation are shown as a good way for a better identification of the process of accumulating the energy to the soil material.
\end{abstract}

\section{Keywords:}

Sub-surface heat accumulation; Solar heat storage;

Soil mass thermal capacity; Ground heat.

\section{Introduction}

The Sun is an unlimited energy source and harnessing of solar radiation is widely supported by government bodies in many countries across the world. Especially, solar thermal wavelengths have been used for practical solutions for years to get alternatives for common heating systems. Even though, the principle of collection of this type of energy is very simple, there are many difficulties to store the gained heat. Using a huge water tank for this purpose means that the final system is very expensive. The natural soil mass that surrounds each house and is pay-free seems to be a good alternative to water tank. The idea to create a cheap thermal energy storage system using ground mass is not brand new and these accumulation systems are successfully used in many countries (ISOMAX, Drake Landing Solar Community, Canada) [4], [5]. The efficiency also depends on the way, how the heat can be distributed throughout the borehole exchangers to the surrounding soil. Measuring of temperature combined with numerical simulation is a good way to identify this process. [1], [2], [3].

\section{Description of the numerical model}

A body of ground with the dimensions of $10 \mathrm{~m} \times 10 \mathrm{~m}$ and depth of $5 \mathrm{~m}$ was defined to analyze the way how the thermal energy is distributed throughout the soil. Nine boreholes stretched to the depth of $2.5 \mathrm{~m}$ with $20 \mathrm{~m}^{2}$ total contact area with surrounding soil are used to transfer heat from solar system to the ground via liquid medium (Fig.1). The soil accumulation volume is thermally insulated on top and sides only. 


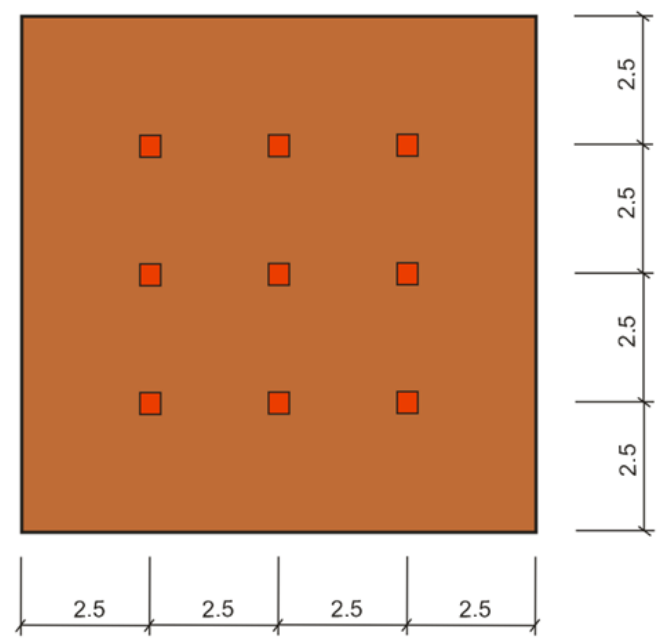

Fig. 1: Boreholes distribution.

The 3D hexahedral conduction elements are used to create FEM model, which can be degenerated into prismatic or tetrahedral elements by collapsing a face, or a face and edge respectively. The element matrices are integrated numerically by Gauss integration with orders of 2 in each local coordinate direction.

Material characteristics of the soil are defined by the thermal capacity of $c=920 \mathrm{~J} \mathrm{~kg}^{-1} \mathrm{~K}^{-1}$, thermal conductivity of $\lambda=0.7 \mathrm{Wm}^{-1} \mathrm{~K}^{-1}$ and the density is $\rho=1800 \mathrm{~kg} \cdot \mathrm{m}^{-3}$. The theoretical capacity of the soil storage system is $138 \mathrm{kWh}^{\circ} \mathrm{C}^{-1}$, if the active height is $3 \mathrm{~m}$.

The solar system is designed for the conditions in central Europe, where an average solar radiation is expected to be $1680 \mathrm{Wm}^{-2}$ per day. The designed area of the solar collector is $20 \mathrm{~m}^{2}$ and the efficiency of collecting and transporting of the heat energy is assumed to only 50 percent. The intensity of heat flux per second is defined for a square meter as a loading for contact area between the surrounded soil and boreholes exchangers. The change of intensity of heat flux is shown in the Fig. 2.

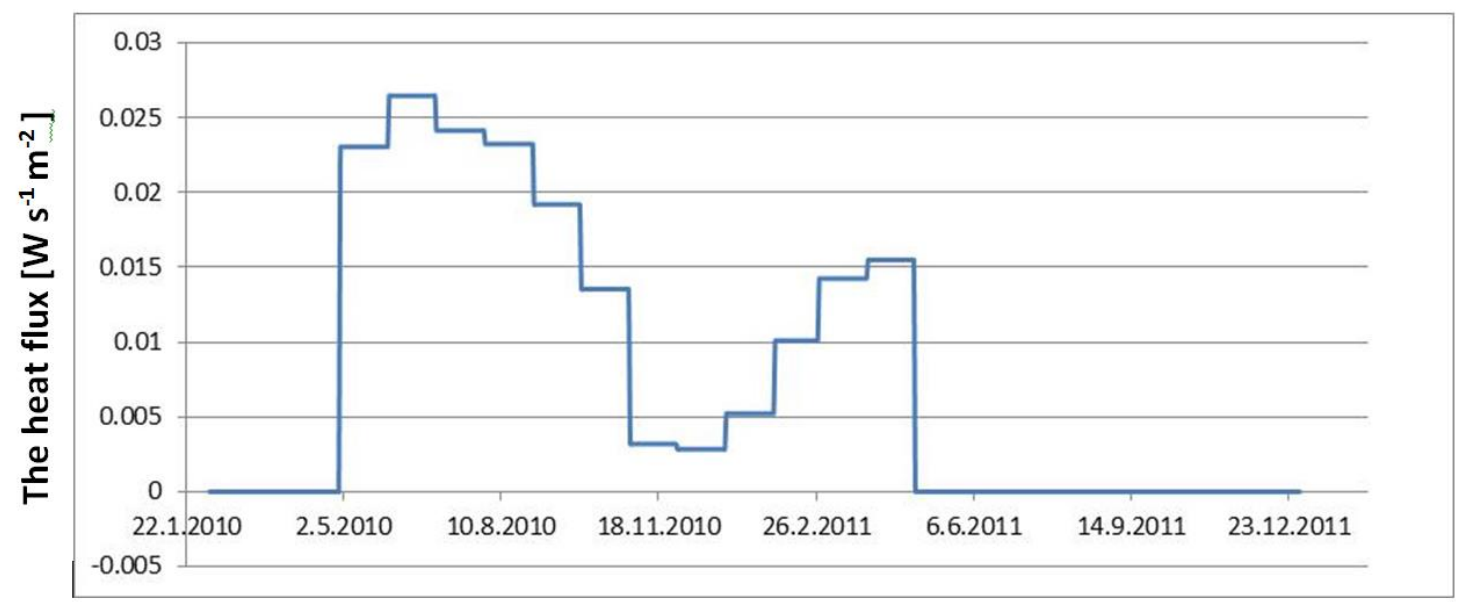

The date of the Day

Fig. 2: The heat flux loading defined for boreholes contact surface.

The boundary temperature along the top of an accumulator is $18{ }^{\circ} \mathrm{C}$ to simulate the placement of this accumulator beneath a house. The temperature defined at the edge of the soil simulates the real conditions reflecting the changes of open air temperature during the year. The boundary temperature also depends on depth. The real measured data was used for simulation (Fig.3). 
The simulation starts in January with zero temperature in the whole modeled space. The beginning of the storage of the thermal energy starts in May. The time between January and May is defined for consolidation of the temperature distribution throughout the soil. Then, the charging of the system starts and continues till September. The whole simulation finishes in March of the following year, because the information about remaining temperature distribution is also important.

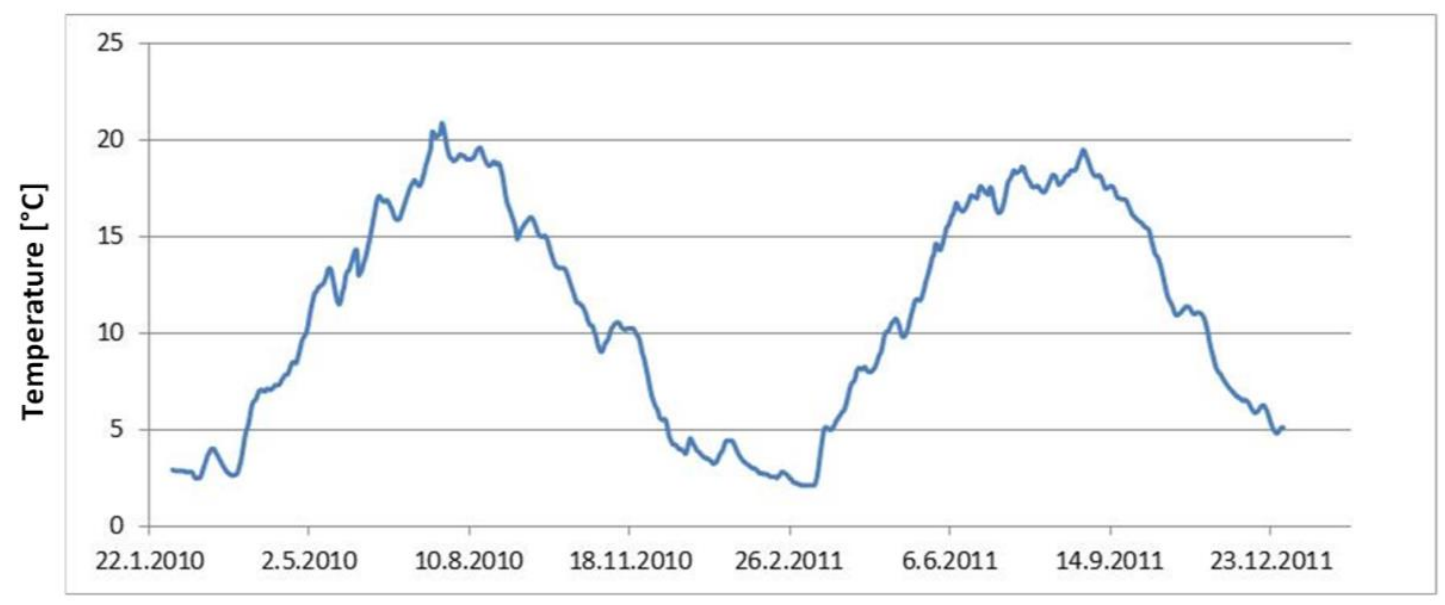

The date of the Day

Fig. 3: The change of temperature in the $0.75 \mathrm{~m}$ deep soil under the surface per year.

\section{Evaluation of results}

The temperature distribution over the whole space is used to obtain results for comparison of changes between the state at the beginning of collection of thermal heat and at the end (Figs. 4, 5, 6). At the beginning, the maximum temperature is $18{ }^{\circ} \mathrm{C}$. At the end of heat charging in September, the maximum temperature reaches $75^{\circ} \mathrm{C}$.
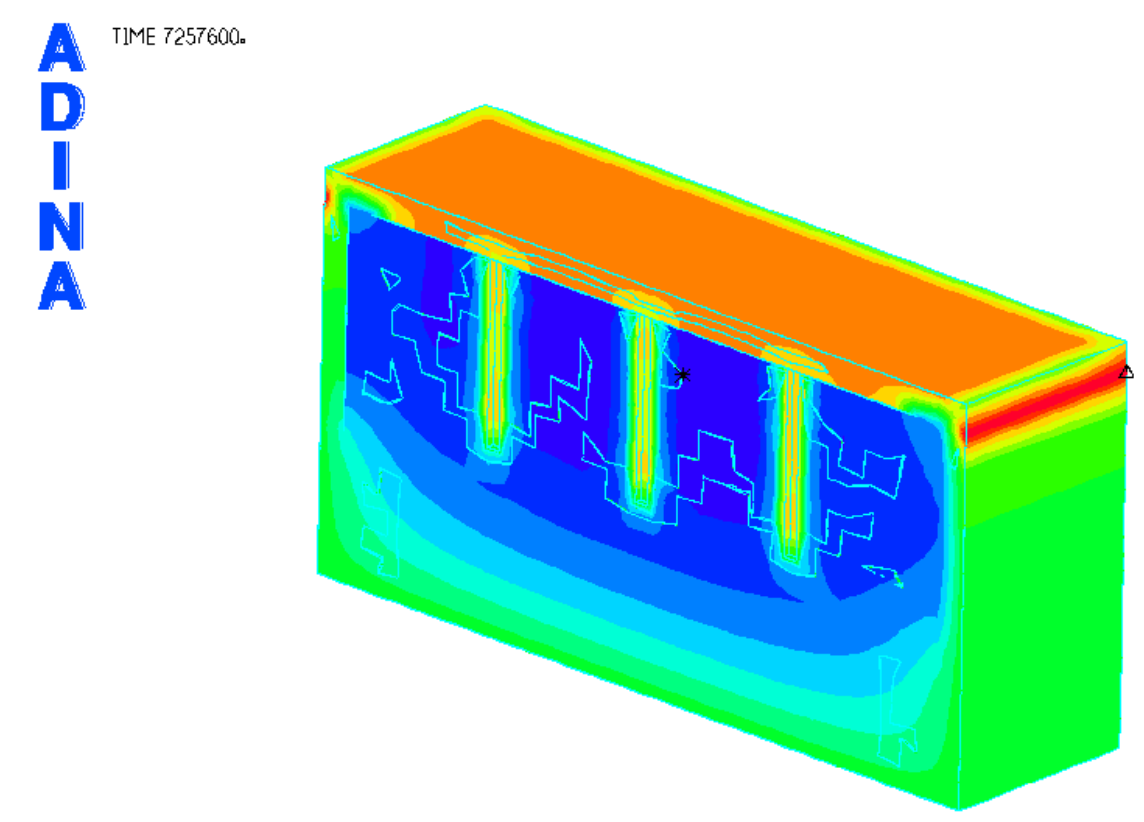

TEMPERATURE TIME 7257600.

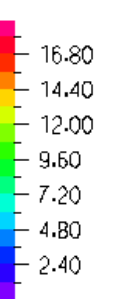

MAXIMUM $\triangle 17.75$
NODE 3087 MINIMUM * 1.471 NODE 10879 (1.493)

Fig. 4: The beginning of the collection of the solar thermal energy in May. 

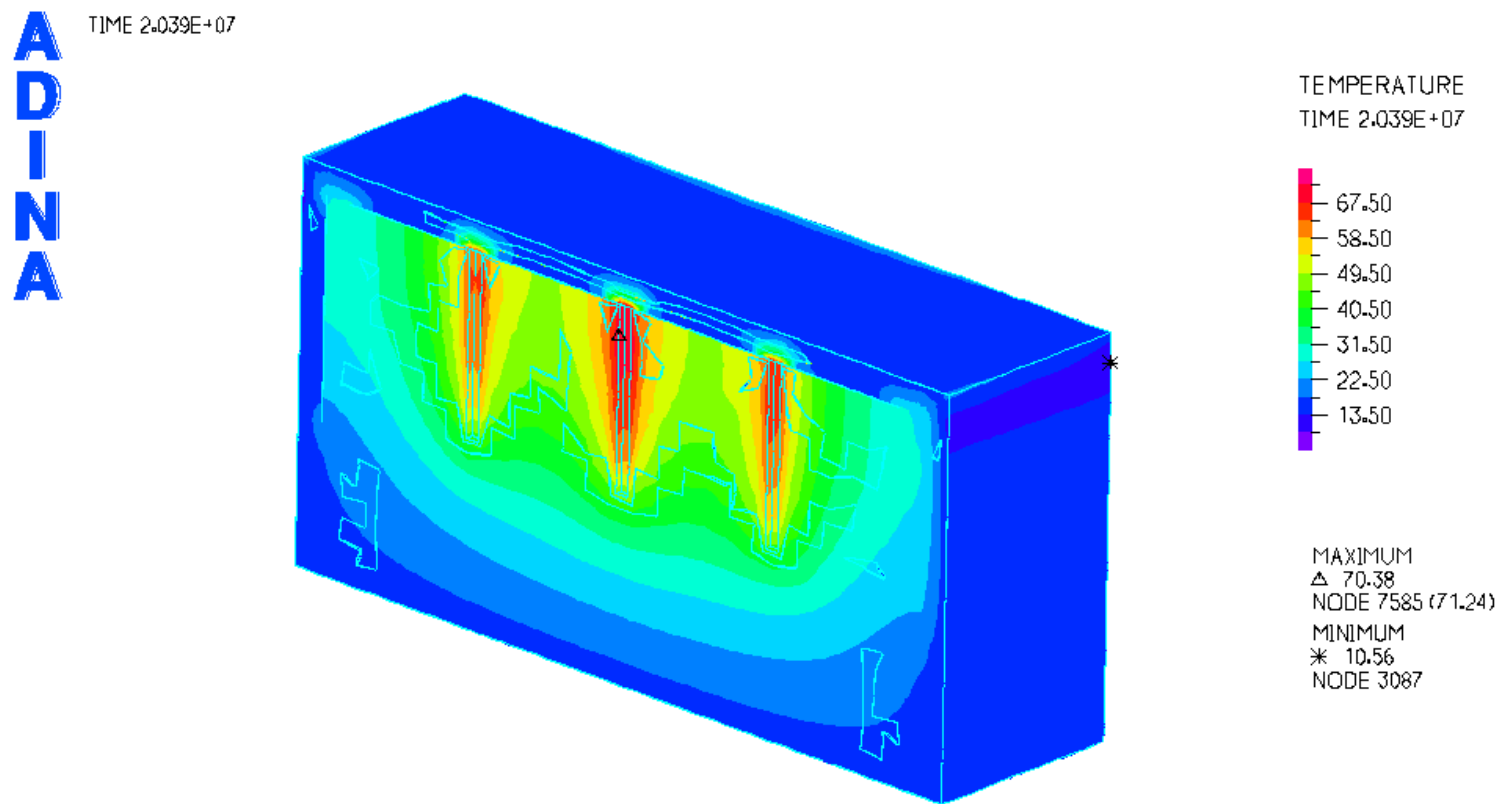

Fig. 5: The end of the collection of the solar thermal energy in September.

The average temperature in the system is $35{ }^{\circ} \mathrm{C}$ in January. It means that there is approximately $1380 \mathrm{kWh}$ stored in the form of heat if the low temperature of $20{ }^{\circ} \mathrm{C}$ is expected as the boundary for the effective usage of the system.

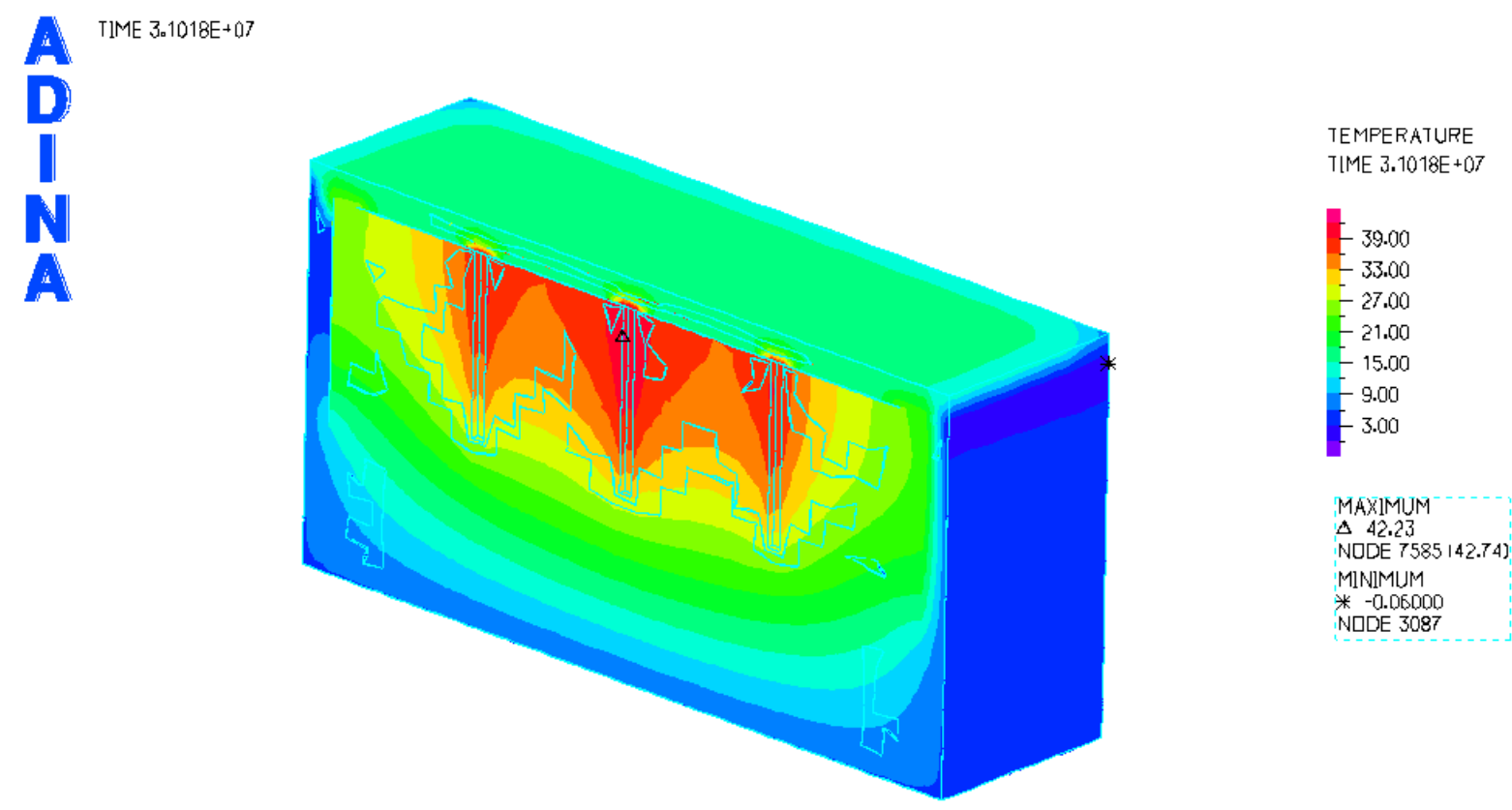

Fig. 6: The final state of the soil heat exchanger in the middle of winter season in January. 


\section{Conclusions}

The results of the numerical simulation showed that the major part of collecting of thermal energy was successfully stored in the underground soil heat exchanger. The location of the nine boreholes seems to be good for equally distributed temperature across the modeled space.

\section{Acknowledgement}

The research is supported by the European Regional Development Fund and the Slovak state budget for the project "Research Centre of University of Žilina", ITMS 26220220183.

\section{References}

[1] Numerical study of natural convection from a heat generating element using a locally divergence free FEM and comparison with experiment, M.R. Rajkumar, G. Venugopal, S. Anil Lal, International Communications in Heat and Mass Transfer, Volume 39, Issue 4, April 2012, Page 530-536.

[2] Transient thermal mechanical analyses using a face-based smoothed finite element method (FSFEM), S.Z. Feng, X.Y. Cui, G.Y. Li, International Journal of Thermal Sciences, Volume 74, December 2013, Pages 95-103.

[3] Determination of temperature distribution in the cutting zone using hybrid analytical-FEM technique, W. Grzesik, International Journal of Machine Tools and Manufacture, Volume 46, Issue 6, May 2006, Pages 651-658.

[4] http://www.dlsc.ca/solar_collect.htm.

[5] http://stavebnictvo.sk/m/blogpost?id=6282648\%3ABlogPost\%3A65924. 